University of South Carolina

Scholar Commons

$8-11-2006$

\title{
Positive Current Correlations Associated with Super-Poissonian Shot Noise
}

Yuanzhen Chen

University of South Carolina - Columbia

Richard A. Webb

University of South Carolina - Columbia, webbra@mailbox.sc.edu

Follow this and additional works at: https://scholarcommons.sc.edu/phys_facpub

Part of the Physics Commons

\section{Publication Info}

Published in Physical Review Letters, Volume 97, Issue 6, 2006, pages 066604-1-066604-4.

Chen, Y. and Webb, R.A. (2006). Positive Current Correlations Associated with Super-Poissonian Shot Noise. Physical Review Letters, 97(6), 066604-1 - 066604-4. doi: 10.1103/PhysRevLett.97.066604 (C) 2006 The American Physical Society.

This Article is brought to you by the Physics and Astronomy, Department of at Scholar Commons. It has been accepted for inclusion in Faculty Publications by an authorized administrator of Scholar Commons. For more information, please contact digres@mailbox.sc.edu. 


\title{
Positive Current Correlations Associated with Super-Poissonian Shot Noise
}

\author{
Yuanzhen Chen* and Richard A. Webb \\ Department of Physics and USC NanoCenter, University of South Carolina, Columbia, South Carolina 29208, USA
}

(Received 20 February 2006; published 11 August 2006)

\begin{abstract}
We report on shot noise cross spectrum measurements in a beam splitter configuration. Electrons tunneling through potential barriers are incident on a beam splitter and scattered into two separate channels. Such a partition process introduces correlations between the fluctuations of the two currents. Our work has confirmed that the generally expected negative correlations resulted from sub-Poissonian electron sources. More interestingly, positive cross correlations associated with barriers exhibiting superPoissonian shot noise have also been observed. We have found that both positive and negative correlations can be related to the noise properties of the electron source.
\end{abstract}

DOI: 10.1103/PhysRevLett.97.066604

PACS numbers: $72.70 .+\mathrm{m}, 73.23 .-\mathrm{b}, 73.40 . \mathrm{Gk}$

In a tabletop Hanbury-Brown-Twiss (HBT) experiment [1-3], the intensities of light beams transmitted through and reflected at a beam splitter are measured by two detectors and then cross correlated in the time domain. The result is a function of the time delay $\tau$ between the two detectors. If a thermal light source is used, a positive correlation at $\tau=0$ is observed. This is interpreted as a bunching effect for photons [2-4]: because of the Bose-Einstein statistics, photons tend to occupy the same state, which leads to a simultaneous arrival of photons at both detectors, thus the positive correlation. On the contrary, electrons obey Fermi-Dirac statistics and cannot occupy the same state; thus, an antibunching effect is expected. As a result, a negative correlation should be observed if electrons instead of photons are used in the HBT experiment [5-10]. This has been recently demonstrated for free electrons [11] and for electrons in a solid state environment [12-15]. In general, the outcome of a cross correlation measurement is determined by the statistical properties of the particle source used. In this Letter, we report on shot noise cross spectrum measurements using tunnel barriers as an electron source. The tunable statistics (noise) of the current emitted by these barriers gives rise to new results for a cross spectrum measurement. In particular, we have made the observation of a positive cross spectrum resulting from super-Poissonian shot noise rather than a bunching effect. Our data show a clear linear relationship between the Fano factor of the electron source and the cross spectrum.

The samples used in this experiment were fabricated in a GaAs/AlGaAs heterostructure with an electron mobility of $6.1 \times 10^{5} \mathrm{~cm}^{2} / \mathrm{V} \mathrm{s}$ and a carrier density of $1.8 \times$ $10^{15} \mathrm{~m}^{-2}$. Metal gates were deposited on top of the sample about $50 \mathrm{~nm}$ above the two-dimensional electron gas (2DEG). A schematic of the gates is given in Fig. 1(a). Negative voltages are applied to gates 1 and 2 (or 3 ) to form a tunnel barrier. With negative voltages applied, gates 6,7 , and 8 together form a beam splitter. Its transmission coefficient can be adjusted by changing the voltage on gate 7 . Electrons are injected from reservoir $A$ (or $B$ ). After tunneling through the barrier, they are incident on the beam splitter, where partition occurs and the electrons are scattered into two channels $C$ and $D$. The current fluctuations in both channels are measured by two cryogenic amplifiers, and are further amplified at room temperature and eventually fed into a spectrum analyzer, which calculates the cross spectrum. All measurements are done in a $20 \mathrm{kHz}$ window around $220 \mathrm{kHz}$ and at a temperature of $70 \mathrm{mK}$ [16].

With such a setup, in an ideal case where all electrons tunneling through the barrier are incident on the beam splitter, theory has predicted [5] that the cross spectrum of the currents in channels $C$ and $D$ should be

$$
S_{C D}=-2 e \operatorname{ITt}(1-t),
$$

where $T$ and $t$ are the transmission coefficients of the tunnel barrier and the beam splitter, respectively. $I$ is the tunneling current (in a range of $100 \mathrm{pA}$ to $2 \mathrm{nA}$ in our measurements). The negative sign is a consequence of the Pauli exclusion principle.
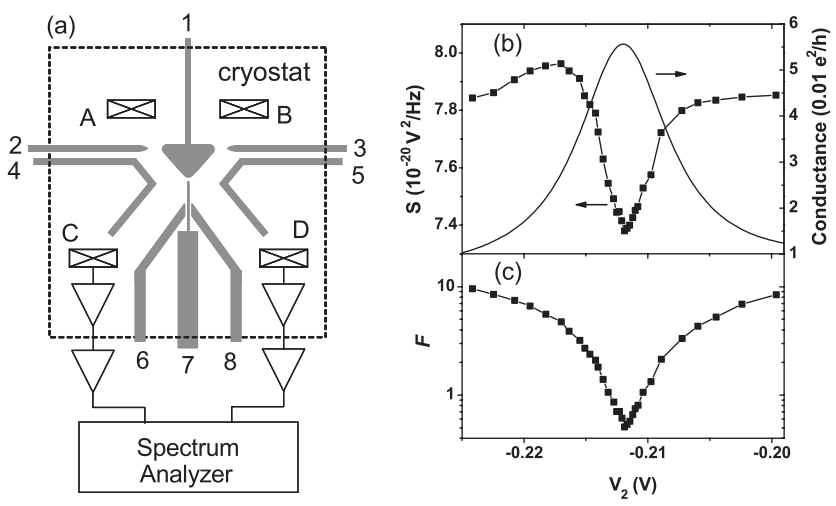

FIG. 1. (a) Schematic of the sample. $A, B, C$, and $D$ are electron reservoirs. High negative voltages are applied to gates 6 and 8 so that the partition of electrons occurs only at the section of gate 7 between the bottom of gate 1 and the top of gates 6 and 8. (b) Conductance and raw data of voltage fluctuations across the loading resistor as a function of the voltage on gate 2. (c) $F$ extracted from (b) using the method in Ref. [16]. 
We note that Eq. (1) has been tested only for ideal tunnel barriers [14,15], where the transport mechanism is direct tunneling with a probability of $T$. This stochastic process leads to a shot noise of $S_{I}=2 e I(1-T)$ in the tunneling current. The Fano factor of such a barrier is then $F=$ $S_{I} / 2 e I=1-T$. Equation (1) can thus be rewritten in the following form, for ideal tunnel barriers:

$$
S_{C D}=2 e I(F-1) t(1-t) .
$$

This equation establishes a relation between the noise property of the source and the resulting cross spectrum.

While the generalization of Eq. (1) to Eq. (2) is straightforward for ideal tunnel barriers, we want to point out that most barriers fabricated in a $2 \mathrm{DEG}$ in GaAs/AlGaAs heterostructures are nonideal [17,18], especially in the pinch-off regime $(T \ll 1)$ [16]. In these barriers, the relation between $F$ and $T$ can be very complicated and extremely sensitive to microscopic details such as the spatial and energy distribution of localized states. As a result, two barriers with the same apparent $T$ can have very different $F$ values. In such a case, Eqs. (1) and (2) disagree with each other. A more striking case is for barriers exhibiting superPoissonian shot noise $(F>1)[17,19,20]$. While for all tunnel barriers one has $T \geq 0$, thus a negative cross spectrum always results according to Eq. (1), Eq. (2) predicts a positive result for the $F>1$ case. To our knowledge, measurements under such a condition $(F>1)$ have never been performed before. In addition, in the sub-Poissonian regime $(F<1)$, only ideal tunnel barriers have been used as a source [12-15]. In the following, we present our systematic study on the relation between $S_{C D}$ and $t$ and $F$ using arbitrary tunnel barriers as a source. Our data show that Eq. (2) is a more general result that can be applied to both ideal and nonideal barriers. We also note that this equation has recently been derived using a Langevin approach [21].

We first characterize the source barrier by measuring its conductance $G$ and $F$ as a function of the gate voltage. In our setup, shot noise signal is loaded to a resistor and measured as voltage fluctuations across it [16]. A circuit model that relates such fluctuations to the shot noise signal is then used to extract $F$ [22]. For an ideal tunnel barrier containing no localized states, $G$ decreases monotonically in the pinch-off region $(T<1)$ as the gate voltage changes to more negative values [16]. Correspondingly, $F$ increases monotonically from 0 to 1 [16]. In most cases, however, deviations of both $G$ and $F$ from those of an ideal tunnel barrier were observed, with one example given in Fig. 1. As reported in Ref. [16], the deviation of $F$ from that of an ideal tunnel barrier always correlates with a nonmonotonical conductance, which is usually caused by localized states. Therefore $F$ shown in Fig. 1(c) is also a consequence of such states. In our measurements, an $F$ between 0.3 and 14 has been observed on 25 barriers. Usually both shot noise suppression and enhancement are observed in the same device as the gate voltages are varied. This offers a tunable noise source for the cross spectrum measurements.

Once the source barrier has been characterized, we then set $V_{2}$ (or $V_{3}$ ) to the value where the barrier has the desired Fano factor and measure $S_{C D}$. Figures 2(a) and 2(b) show $S_{C D}$ as a function of the beam splitter voltage $V_{7}$ for barrier 1 -A at two different $F$ values. For $F=0.6, S_{C D}$ shows a negative dip around $V_{7}=-162 \mathrm{mV}$, which qualitatively agrees with the general expectation for a negative cross spectrum for electrons in mesoscopic conductors [5,1215]. Quantitatively, Eq. (1) predicts a minimum of -0.0059 (normalized to $2 e I$ ) at $t=0.5$ for $F=0.6$, whereas Eq. (2) predicts a minimum of -0.1 . The measured minimum in Fig. 2(a) is -0.08 , in better agreement with Eq. (2). The most interesting feature, however, is the prominent positive peak that also appears at $V_{7}=$ $-162 \mathrm{mV}$ in Fig. 2(b). For $F=6$, Eq. (1) predicts a minimum of -0.00175 , which completely disagrees with the measurement, a maximum of 1 . On the other hand, Eq. (2) predicts a maximum of 1.25 that describes the data reasonably well.

According to Eq. (2), $S_{C D}$ has a maximum absolute magnitude at $t=0.5$ for a given $F$. In order to relate the voltage $V_{7}$ to $t$, an independent dc transport measurement is performed to obtain the resistance $R$ of the beam splitter as a function of $V_{7} . t\left(V_{7}\right)$ is then extracted from $R\left(V_{7}\right)$ using the Landauer-Büttiker formula [23], $R=\left(h / 2 e^{2}\right) \times$ $(1-t) / t$. We found that $t=0.46$ at $V_{7}=-162 \mathrm{mV}$ where the measured $S_{C D}$ shows a dip (peak) in Fig. 2(a) [Fig. 2(b)], being very close to the expected value of 0.5 .

We performed similar measurements on 12 barriers at various $F$ values. Each measurement yielded a $S_{C D}$ similar to that in Figure 2(a) or 2(b). We then extracted the minimum (maximum) of $S_{C D}(t=0.5)$ for cases like Fig. 2(a) [2(b)] and studied the correlation between these values and $F$, as shown in Fig. 3, for four barriers. It is obvious that for all four barriers $S_{C D}$ is always negative for $F<1$ and positive for $F>1$. The solid lines in Fig. 3 are linear fits to the data. The slope of these lines varies in a range between 0.18 and 0.21 . According to Eq. (2), the

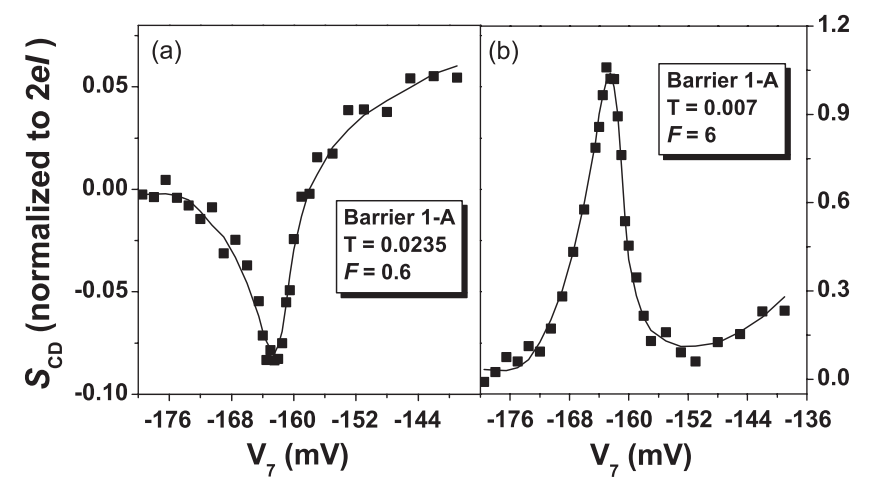

FIG. 2. Measured cross spectrum (squares) as a function of the beam splitter voltage for the same source barrier with two different $F$ values. Solid lines are guides for the eye. 


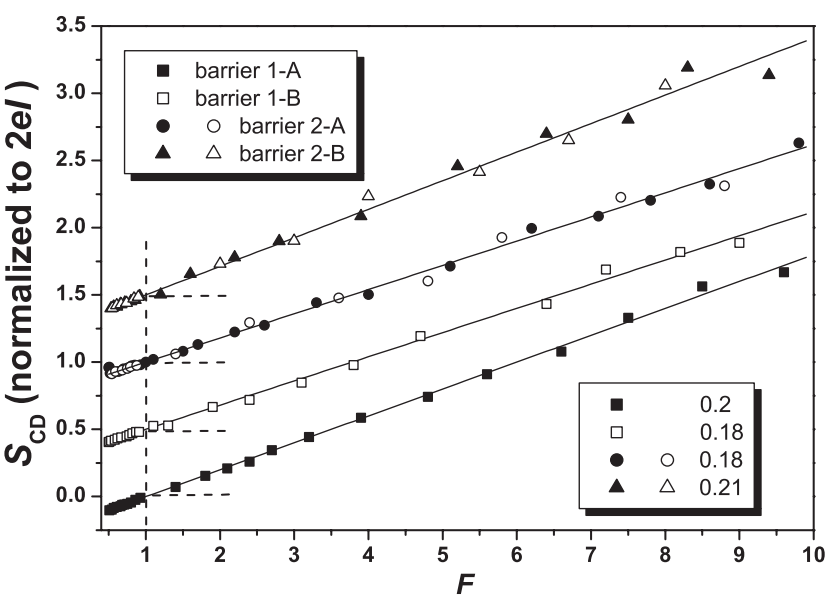

FIG. 3. Measured maximum $S_{C D}$ as a function of $F$ for four different barriers. Data for barriers 1-B, 2-A, and 2-B are shifted relative to that for $1-\mathrm{A}$ by $0.5,1.0$, and 1.5 units, respectively. Horizontal dashed lines indicate the position of $S_{C D}=0$ for each barrier. Vertical dashed line marks $F=1$. Solid lines are linear fits to data, with slopes given in the lower right inset.

maximum magnitude of $S_{C D}$ has a form of $0.25(F-1)$ (normalized to $2 e I$ ), which gives a slope value of 0.25 , in reasonable agreement with the experimental data. In order to explain the difference, we note that both Eqs. (1) and (2) are valid only for ideal cases where all electrons emitted by the source are incident on the beam splitter. This requirement is not satisfied in our samples. For example, part of the tunneling electrons can go directly to reservoir $C$ (if current is injected by reservoir $A$ ) without any scattering at the beam splitter, thus reducing $S_{C D}$. Figure 4(a) supports this explanation by comparing the data in Fig. 2(a) to a similar measurement but with $V_{4}=0 \mathrm{mV}$. The magnitude of $S_{C D}$ decreases significantly as a result of setting $V_{4}$ to zero. Gate 4 basically serves as a guide for electrons from the source barrier to the beam splitter. When $V_{4}$ is set to zero, electrons tunneling through the source barrier are not

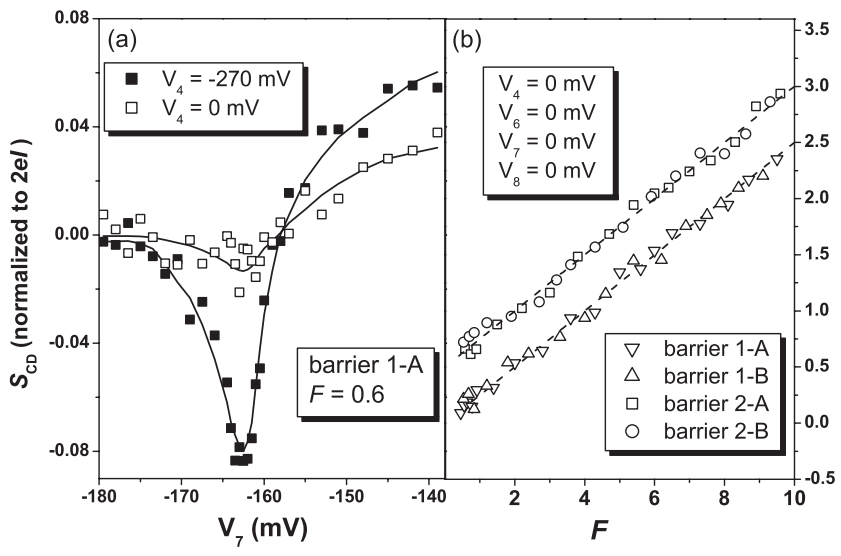

FIG. 4. (a) $S_{C D}$ as a function of the beam splitter voltage with different $V_{4}$ values; solid lines are guides for the eye. (b) $S_{C D}(F)$ with the beam splitter wide open for 4 different barriers; for clarity, the data for barrier 2-A and 2-B are shifted up by 0.5 unit. well directed to the beam splitter. Most electrons go to the reservoir at the same side of the source barrier directly and do not reach the beam splitter. Therefore the cross spectrum is highly reduced.

Similar data analysis can also be used for data taken at $t$ values different from 0.5 . We have found reasonable agreement between our data and Eq. (2) for $0.2<t<0.7$. Outside this range, there is less agreement, which is probably due to the reduced cross spectrum signal (thus the measurement accuracy). In some samples, localized states near the beam splitter at $t<0.2$ were observed, which may also cause a deviation from Eq. (2).

All our data are reproducible when gate voltages are set to zero and reapplied, so long as the sample is kept at low temperatures. After thermal cycling to room temperature and cooling back down to low temperatures, the source characterization [Figs. 1(b) and 1(c)] usually changes. For example, both the position and magnitude of the peak of $F$ may vary. The changes are not predictable and different from barrier to barrier. We believe they are indications of the rearrangement of localized states in space and energy configuration. On the other hand, for a given sample the $S_{C D}$ vs $F$ relation does not change after thermal cycling (e.g., the open circles and open triangles in Fig. 3). In other words, the same source fluctuations always lead to the same cross spectrum.

Our data strongly support Eq. (2). In particular, the sign of $S_{C D}$ is determined by $F$ of the electron source. The observed positive cross spectrum here is a consequence of the source emitting electrons with a super-Poissonian statistics $(F>1)$. This should be distinguished from positive correlations with other origins. One example is given by the positive $S_{C D}$ observed at $V_{7} \geq-152 \mathrm{mV}$ in both Figs. 2 and 4(a). In that range of $V_{7}$, the resistance of the beam splitter is much less than $h /\left(2 e^{2}\right)$. In other words, $t \sim$ 1. Equation (2) predicts $S_{C D} \rightarrow 0$ as $t \rightarrow 1$, while in both Figs. 2 and 4(a) $S_{C D}$ becomes positive and keeps increasing as $V_{7}$ becomes less negative. Eventually, at $V_{7}$ higher than $-110 \mathrm{mV}, S_{C D}$ saturates at a positive value [not shown in Figs. 2 and 4(a)]. To understand this positive cross spectrum, we set the voltages on gates $4,5,6,7$, and 8 to zero and measure $S_{C D}$ as a function of $F$. Figure 4(b) shows the results for four different barriers. For clarity, the data of 2A(B) are shifted up by 0.5 units. In such a measurement, $S_{C D}$ is still a linear function of $F$ but is always positive for all $F$ values, in contrast with the data in Fig. 3. This can be explained in the following way. When the lower part of the sample is wide open, there is no partition process and electrons are quickly thermalized in the reservoir (now $C$ and $D$ are joined together). The potential of this reservoir fluctuates due to the noise in the source current. Therefore the situation is equivalent to a fluctuating source (the reservoir here) driving two classical resistors (the input impedance of the cryogenic amplifiers), which always leads to a positive correlation. With this model, the cross spectrum can be easily calculated, shown as dashed lines in Fig. 4(b), in very good agreement with the experimental 
data. We believe the positive spectrum at $V_{7} \geq-152 \mathrm{mV}$ in Figs. 2 and 4(a) has the same origin. For example, from independent measurements, we know that at $V_{7}>$ $-110 \mathrm{mV}$ for barrier $1-\mathrm{A}$, the beam splitter is already wide open. However, a quantitative description of the transition area (e.g., from around -152 to $-110 \mathrm{mV}$ ) requires more detailed information on the scattering of electrons at a beam splitter with $t \sim 1$.

A positive cross spectrum has also been predicted [24] and recently observed by Oberholzer et al. [25]. In their work, a positive cross correlation results from a fluctuating reservoir injecting electrons in a correlated way into two edge channels in the quantum Hall regime, where there is no super-Poissonian noise source present. Such a result is similar to our $F<1$ data shown in Fig. 4(b).

We also want to point out that the observed positive cross spectrum discussed in this Letter is not a demonstration of a bunching effect, but rather a consequence of a super-Poissonian electron source. Because of the Fermi statistics, common electron sources usually exhibit subPoissonian shot noise, or $F \leq 1$. Under certain conditions (e.g., a tunnel barrier containing interacting localized states), a source can emit electrons in a modulated pattern [17], like the on-off modulation observed in the wellknown random telegraph noise [26]. This can lead to super-Poissonian fluctuations $[17,26]$. When electrons emitted by such a source are scattered at a beam splitter, the two resulting currents exhibit fluctuations in a modulated pattern similar to that of the source current. Therefore a positive correlation between them should be expected. However, there is a profound difference between the positive result in this case and that observed in an HBT experiment. In the latter, the positive sign comes from the fact that photons travel in a bunching manner and is due to the intrinsic quantum statistical properties of photons. On the other hand, bunching simply does not exist for electrons unless they are in the form of entangled spin pairs [27]. Experimentally speaking, the relatively low frequency cross spectrum measurements done in this Letter are similar to a time domain cross correlation measurement with a large time delay $\tau$. If a high frequency measurement is performed, which is equivalent to a time domain measurement with $\tau$ comparable to the single particle passage time $\tau_{p}$, a negative correlation should be recovered. In the model of super-Poissonian noise caused by modulations with a characteristic time $\tau_{m}\left(\tau_{m} \gg \tau_{p}\right)$, our simulation [28] shows that even at a frequency as low as $1 / \tau_{m}$, the cross spectrum becomes negative. The positive cross spectrum observed here is simply an indication that the mechanism leading to the super-Poissonian noise has a characteristic frequency $\left(1 / \tau_{m}\right)$ much higher than that used in our measurement, namely, $220 \mathrm{kHz}$.

In summary, we have performed shot noise cross spectrum measurements in a beam splitter configuration using tunnel barriers as electron sources. The observed cross spectrum is closely related to the noise properties of the source, and can be reasonably well described by Eq. (2). Tunnel barriers with $F<1$ always yield a negative cross spectrum, which is consistent with existing work. However, the spectrum usually has a very different magnitude from that for an ideal tunnel barrier. On the other hand, a positive cross spectrum always results for barriers exhibiting super-Poissonian shot noise. To our knowledge, this is the first demonstration of a positive correlation associated with a super-Poissonian electron source.

We want to thank Markus Büttiker for very helpful and stimulating discussions. This work was supported by NSF through Grant No. DMR0439137.

*Now at Department of Physics and Astronomy, University of Pennsylvania, Philadelphia, PA 19104, USA.

[1] R. Hanbury-Brown and R. Q. Twiss, Philos. Mag. 45, 663 (1954).

[2] R. Hanbury-Brown and Q. R. Twiss, Nature (London) 177, 27 (1956).

[3] B. L. Morgan and L. Mandel, Phys. Rev. Lett. 16, 1012 (1966).

[4] H. Paul, Rev. Mod. Phys. 54, 1061 (1982).

[5] Ya. M. Blanter and M. Büttiker, Phys. Rep. 336, 1 (2000).

[6] M. Büttiker, Phys. Rev. Lett. 65, 2901 (1990).

[7] T. Martin and R. Landauer, Phys. Rev. B 45, 1742 (1992).

[8] M. Büttiker, Phys. Rev. B 46, 12485 (1992).

[9] R. C. Liu and Y. Yamamoto, Phys. Rev. B 49, 10520 (1994).

[10] E. V. Sukhorukov and D. Loss, Phys. Rev. Lett. 80, 4959 (1998).

[11] H. Kiesel et al., Nature (London) 418, 392 (2002).

[12] R. C. Liu et al., Nature (London) 391, 263 (1998).

[13] W. D. Oliver et al., Science 284, 299 (1999).

[14] M. Henny et al., Science 284, 296 (1999).

[15] S. Oberholzer et al., Physica (Amsterdam) 6E, 314 (2000).

[16] Y. Chen and R.A. Webb, Phys. Rev. B 73, 035424 (2006).

[17] S. S. Safonov et al., Phys. Rev. Lett. 91, 136801 (2003).

[18] S.H. Roshko et al., Physica (Amsterdam) 12E, 861 (2002).

[19] G. Iannaccone et al., Phys. Rev. Lett. 80, 1054 (1998).

[20] V. V. Kuznetsov et al., Phys. Rev. B 58, R10159 (1998).

[21] M. Büttiker (private communication).

[22] The circuit model was discussed in some detail in Ref. [16] [e.g., see Eq. (4) in Ref. [16] and related discussion].

[23] Y. Imry, Introduction to Mesoscopic Physics (Oxford University Press, New York, 1997).

[24] C. Texier and M. Büttiker, Phys. Rev. B 62, 7454 (2000).

[25] S. Oberholzer et al., Phys. Rev. Lett. 96, 046804 (2006).

[26] M. J. Kirton and M. J. Uren, Adv. Phys. 38, 367 (1989).

[27] G. Burkard et al., Phys. Rev. B 61, R16303 (2000).

[28] Y. Chen and R. A. Webb (to be published). 